\title{
miRNA-425-5p enhances diffuse large B cell lymphoma growth by targeting PTEN
}

\author{
Weihao Wu ${ }^{1}$, Longtian Chen ${ }^{1}$, Congjie Chen ${ }^{1}$, Lian Yu ${ }^{1}$, Junqiong Zheng ${ }^{2}$ \\ ${ }^{1}$ Department of Hematology, Longyan First Hospital Affiliated to Fujian Medical University, Longyan, China; ${ }^{2}$ Department of Oncology, Longyan \\ First Hospital Affiliated to Fujian Medical University, Longyan, China \\ Contributions: (I) Conception and design: J Zheng, L Yu; (II) Administrative support: W Wu; (III) Provision of study materials or patients: L Chen; (IV) \\ Collection and assembly of data: C Chen; (V) Data analysis and interpretation: C Chen; (VI) Manuscript writing: All authors; (VII) Final approval of \\ manuscript: All authors. \\ Correspondence to: Junqiong Zheng. Department of oncology, Longyan First Hospital Affiliated to Fujian Medical University, Fujian, China. \\ Email: zhengjunqiong2008@126.com; Lian Yu. Department of Hematology, Longyan First Hospital Affiliated to Fujian Medical University, Fujian, \\ China. Email: yulian_ly@126.com.
}

\begin{abstract}
Background: At present, cancer is one of the greatest threats to mankind, and is associated with the highest rates of morbidity and comorbidity. Recently, the advancements in molecular biology have led to an in-depth understanding of the underlying pathophysiology, which may further impact the lead time in the context of early discovery and effective therapy of cancer. Therefore, the present study proposes a better understanding of the role of micro(miR)-425-5p in diffuse large B-cell lymphoma (DLBC).

Methods: qRT-PCR was carried out to detect the relevant proteins, miRNA and mRNA RNA gene expression in DLBC cells. The effect of miR-425-5p on DLBC growth was examined by CCK-8 and colony formation assays. The binding relationship between genes was verified by dual-luciferase reporter gene assay.

Results: We demonstrated how the over-expression of miR-425-5p can lead to increased progression of DLBC by increasing the cellular proliferation rate and colony-forming ability. Additionally, we also found that the expression of miR-425-5p could be significantly inhibited on the basis of phosphatase and tensin homolog (PTEN) signaling pathways.

Conclusions: The present study concludes that miR-425-5p is responsible for the oncogenic progression and relapse of DLBC tumorigenesis via PTEN/PI3K signaling, which can thus be effectively used to achieve better therapeutic outcomes.
\end{abstract}

Keywords: miR-425-5p; diffuse large B cell lymphoma (DLBC); PTEN; PI3K

Submitted Sep 29, 2021. Accepted for publication Nov 24, 2021.

doi: $10.21037 /$ tcr-21-2394

View this article at: https://dx.doi.org/10.21037/tcr-21-2394

\section{Introduction}

The rising incidence of non-Hodgkin's lymphoma has triggered a sudden urgency in the scientific community to explore effective treatment strategies, in order to achieve better therapeutic outcomes. Among the many common forms of non-Hodgkin's lymphoma that are indolent or aggressive is diffuse B-cell lymphoma (DLBC). Rigorous analysis through micro-array-based gene profiling (1) has proposed the identification of two major subtypes: activated B-cell like (ABC) and germinal centre B-cell like carcinoma. Other techniques, such as immunohistochemistry (2), Nanostringn Counter system (3), and Hans classification (4), have supported the obtained outcomes. Studies have also confirmed that effective therapeutic outcomes and rapid prognoses are associated with the identification of mutagenic changes causing structural relocation due to Myc, BCL-2, and BCL-3 (5).

At present, patients with a known diagnosis of DLBC are offered rituximab plus cyclophosphamide, prednisone, 
along with other corticosteroids (such as doxorubicin and vincrinstine). This approach has been found to support quick recovery, allowing for a complete cure in $60-70 \%$ of patients. However, there is a concern for the remaining $30-40 \%$ patients, who reportedly experience relapse (5). Consequently, the present research is intended to understand the biochemical mechanism associated with the initial trigger and rapid progression of DLBCL. Additionally, the analysis will also be conducive to identifying new molecular targets leading to effective prognosis as well as the implementation of better therapeutic modules.

According to previous studies, oncogenic cellular division, migration, and proliferation are extensively regulated by microRNAs (miRNAs) (6-8), which are therefore considered as key players in the development of various forms of lymphatic cancers (including DLBC) (9-12).

According to previous studies, oncogenic cellular division, migration, and proliferation are extensively regulated by microRNAs (miRNAs) (6-8), which are therefore considered as key players in the development of various forms of lymphatic cancers (including DLBC) $(9,10)$.

A large number of studies have found that miRNAs are abnormally expressed in a variety of malignant tumors and are associated with malignant tumor growth (11-13). Expression of miR-425-5p is upregulated in renal cell carcinoma and promotes cell viability, invasion and migration (12). In prostate cancer, high miR-425$5 \mathrm{p}$ expression can significantly promote the proliferation, migration and invasion of tumor cells (13). Previous studies have indicated that miRNA-425-5p-PTEN axis play key role in breast cancer and lung cancer $(14,15)$, however the miRNA-425-5p-PTEN axis in diffuse large B cell lymphoma remain unclear.

Thus, in the present study, we report the effect of miR425-5p up-regulation in the oncogenic development of DLBC, and further suggest that it can be regarded as a novel target to block the expression of miR-425-5p via guided signaling pathways, such as phosphatase and tensin homolog (PTEN)/phosphatidylinositol 3-phosphate kinase (PI3K). We present the following article in accordance with the MDAR reporting checklist (available at https://dx.doi. org/10.21037/tcr-21-2394).

\section{Methods}

\section{Patient specimens}

In total, 25 DLBCL samples and 25 normal samples of adjacent lymph nodes were collected during biopsy from July 2017 to January 2019 at Zhejiang Cancer Hospital who donated DLBC samples, as well as 25 control samples collected from lymphoid hyperplasia patients. This study was approved by the institutional review board of First Hospital Affiliated to Fujian Medical University. The study was conducted in accordance with the Declaration of Helsinki (as revised in 2013). All participants were informed about the outcome and provided written informed consent, and participants identity was also kept hidden.

\section{Analysis of the cell lines, cell culture, and transfection profile}

All the required cell lines, including DLBCL (U2939, TMD8) and human B lymphocyte (GM12878) cell lines were acquired from ATCC (Manassas, VA, USA). Others, like classic Hodgkin's lymphoma (L428), mantle cell lymphoma (Jeko-1), follicular lymphoma (WSU-FSCCL), and DLBCL (OCI-Ly3) cell lines, were obtained from the local cell bank of the Chinese Academy of Sciences. With $10 \%$ fetal bovine serum (FBS) and $100 \mu \mathrm{g} / \mathrm{L}$ penstrep combinations at $5 \%$ (carbon dioxide) $\mathrm{CO}_{2}$, these cell lines were grown in RPMI-1640 medium. DLBCL cell lines (U2939, TMD8) were treated with LY294002, which is a previously described PI3K inhibitor obtained from Sigma Aldrich (Sigma-Aldrich; Merck KGaA). miR425-5p mimic (5'-CTGCUAGAGGCAGCGCTGT-3') and negative control (NC) (termed as NC, 5 ' - A C UACUTGG U GA C GC T GA GCA - 3 ' ) oligonucleotides were purchased from Guangzhou RiboBio Co., Ltd. (Guangzhou, China). In accordance with the manufacturer's protocol, transfection of cells with $50 \mathrm{nM}$ oligonucleotides was carried out using Lipofectamine ${ }^{\circledR} 2000$ (Invitrogen; Thermo Fisher Scientific, Inc., California, USA). Lentiviral vector (LV)-NC and LV-PTEN were purchased from Gene Pharma (Shanghai, China) and transfected into DLBCL cell lines (U2939, TMD8) in strict accordance with the manufacturer's instructions.

\section{PI3K activity assays}

The activity of PI3K was assayed as described earlier (8). PI3 K activity in the immunoprecipitates was analyzed using a PI3K enzyme - linked immunosorbent assay kit (Echelon Biosciences, Salt Lake City, UT, USA) based on the manufacturer's instructions. 


\section{Luciferase assay}

Luciferase activity was detected at $24 \mathrm{~h}$ using a DualLuciferase Reporter Assay kit (cat. no. E1910; Promega Corporation, Madison, WI, USA) and reported as previous (12).

\section{Cell growth assays}

The viability of cells was explored using the Cell Counting Kit-8 (CCK-8) kit, as per the manufacturer's protocol, according to which seeding of approximately 5,000 indicated treated TMD8 and U2932 cells was conducted in 96-well plates and were incubated. Along with the addition of CCK-8, the cells were transfected with the same for $48 \mathrm{~h}$. The absorbance was measured after $1 \mathrm{~h}$ of incubation.

\section{Colony formation assay}

To assess the colony-forming ability of the cells, we seeded approximately 1,000 indicated treated TMD 8 and U2932 cells per well of a $10 \mathrm{~mm}$ culture dish. The cells were maintained in optimum culture conditions for approximately 2 weeks prior to the analysis. After 2 weeks, the staining of cells was carried out with crystal violet (1\%) and the colony number was estimated.

\section{Real-time reverse transcription-PCR (qRT-PCR) analysis}

The qRT-PCR analysis was performed as previous (8). The final expression profile of miR-425-5p was assessed using SYBR PrimeScript Kits (Qiagen, Hilden, Germany) according to the manufacturer's instructions, U6 was used as an internal control for microRNA, and GAPDH was used for mRNA by employing the $2^{-\Delta \Delta C t}$ method. The primer sequences used for the analysis included miR425-5p (F: 5'-GGGGAGTTAGGATTAGGTC-3', and R: 5'-TGCGTGTCGTGGAGTC-3'), U6 (F: 5'-CTCGCTTCGGCAGCACA-3', and R: $5^{\prime}$-AACGCTTCACGAATTTGCGT-3'), PTEN (F: 5'-TGGATTCGACTTAGACTTGACC-3', and R: 5'-AGGATATTGTGCAACTCTGCAA-3'), and glyceraldehyde-3-phosphate dehydrogenase (GAPDH) (F: 5'-CATCACCATCTTCCAGGAGCG-3', and R: 5'-TGACCTTGCCCACAGCCTT-3') (F: forward primer; $\mathrm{R}$ : reverse primer).

\section{Statistical analysis}

Data analyses were conducted using SPSS 19.0 software (SPSS; Inc, Chicago, IL, USA). Controls were compared using Analysis of Variance (ANOVA) (one-way) with Duncan's post-hoc test or the student's $t$-test, along with $\mathrm{P}$ values, which were considered significant at $<0.05$. Taking the average of the three variables, the data were analyzed using mean \pm standard deviation (SD).

\section{Results}

\section{Enhanced expression of miR-425-5p in DLBC}

All the samples obtained from the test as well as controls were assessed for expression profiles of miR-425-5p, using RT-PCR as demonstrated in Figure $1 \mathrm{~A}$ and cells obtained from lymphoma tissues were high in expression in comparison with controls. In addition, the expression profiles were also found to be higher in lymphoma cell lines as compared to their control version like B-lymphocyte cell lines (GM12878) (Figure 1B). The findings are in consistence with previous findings of other cancers, further suggesting significantly enhanced expression of miR-425-5p in DLBC.

\section{Increased proliferation of DLBC cells by miR-425-5p}

The association between an increase in the level of miR-425-5p and increased proliferation rates of cells obtained from DLBC patients was assessed. As shown in Figure $2 A-2 C$, there existed a direct association with increased proliferation rate, and colony-forming capacity (Figure 2D,2E). These results further support that miR-425$5 \mathrm{p}$ enhanced DLBC cells proliferation.

\section{PTEN is targeted by miR-425-5p}

Using Target Scan 7 (https://www.targetscan.org/vert_71/), we observed that PTEN was a predicted miR-425-5p target (Figure $3 A$ ). Considering that inactivation of the PTEN signaling pathway is closely associated with the promalignancy of DLBC (16), we focused on the miR-425-5p/ PTEN axis in DLBC. In reporter assays of PTEN 3'-UTR, there was miR-425-5p-mediated suppression of widetype (WT) PTEN expression (TMD8-WT, mimic NC vs. miR-425-5p mimic: $1.000 \pm 0.178$ vs. $0.397 \pm 0.109, \mathrm{P}<0.001$; 
A

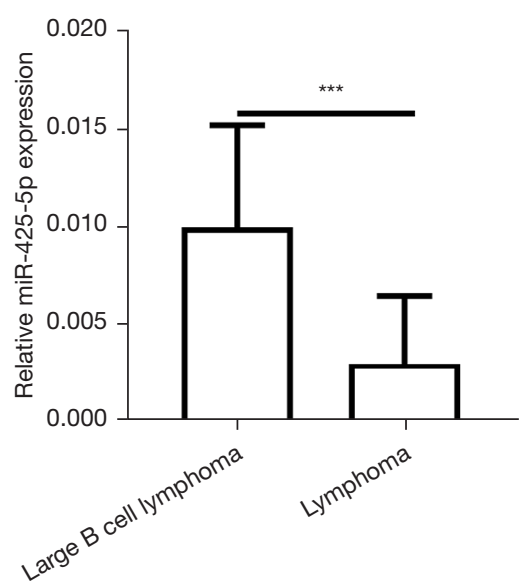

B

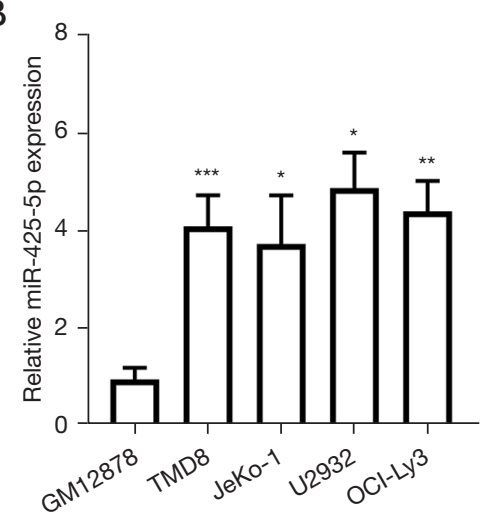

Figure 1 miR-425-5p is highly expressed in DLBC. (A) qRT-PCR of miR-425-5p in 25 DLBC and 25 lymphoma tissues. (B) qRT-PCR of miR-425-5p in human DLBC cell lines and human B lymphocyte (GM12878). Data: mean \pm SD. ${ }^{*}, \mathrm{P}<0.05 ;{ }^{* *}, \mathrm{P}<0.01{ }^{*}{ }^{* *}, \mathrm{P}<0.001$. DLBC, diffuse large B-cell lymphoma; qRT-PCR, real-time reverse transcription-PCR.

A

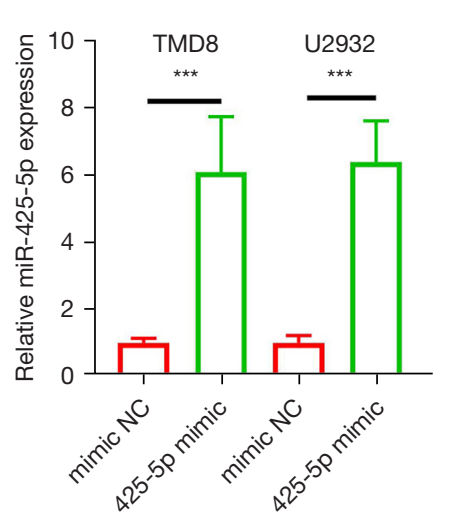

D

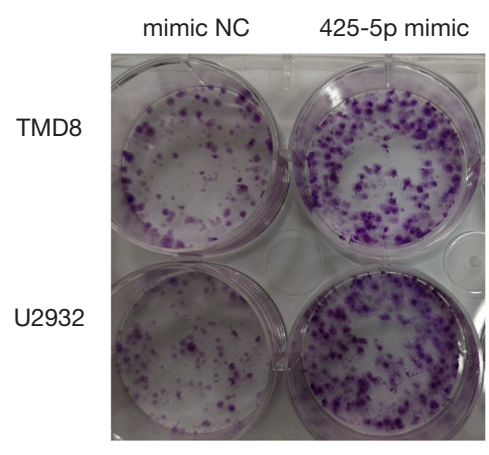

B

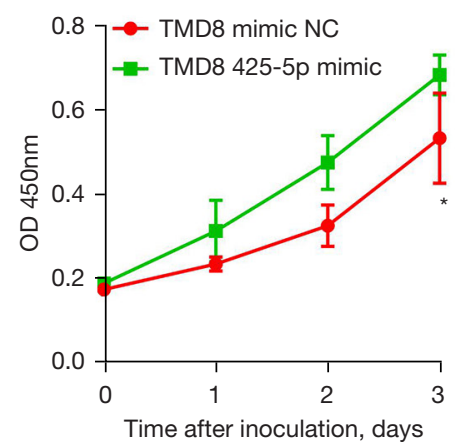

$\mathrm{E}$

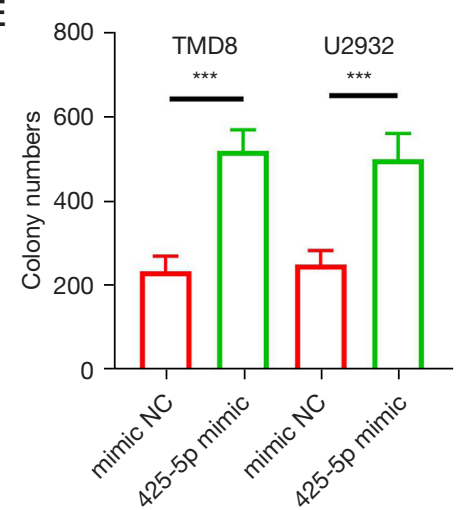

C

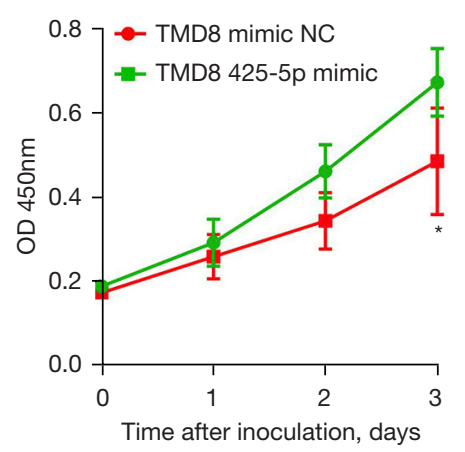

Figure 2 miR-425-5p promotes DLBC. (A) miR-425-5p expression in TMD8 and U2932 cells; (B,C) CCK-8; (D,E) colony-forming assay in TMD8 and U2932 cells with miR-425-5p mimic. Data: mean \pm SD. *, $\mathrm{P}<0.05$; ***, $\mathrm{P}<0.001$. DLBC, diffuse large B-cell lymphoma. 
A

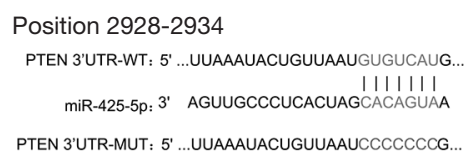

D

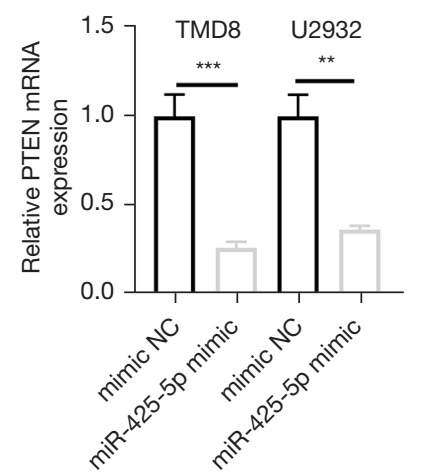

B

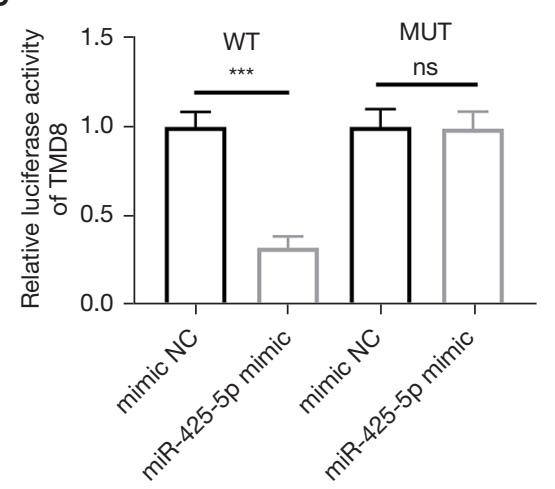

$E$

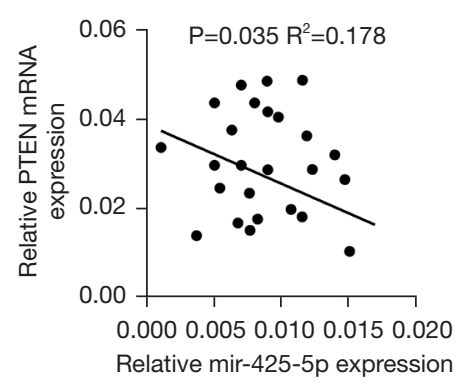

C

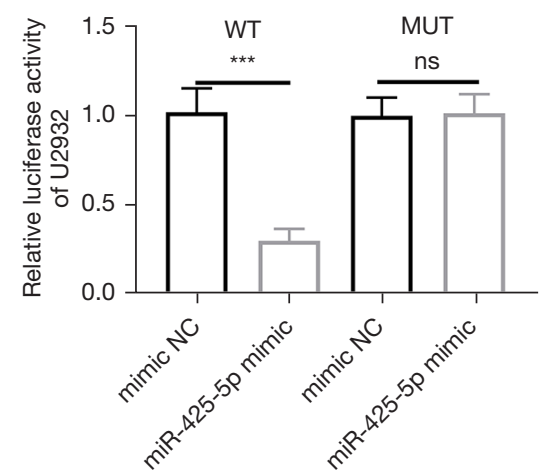

Figure 3 miR-425-5p targets PTEN in DLBC. (A) Predicted binding of miR-425-5p and PTEN; (B,C) relative luciferase activity of PTEN-WT and PTEN-MUT in DLBC cells with miR-425-5p mimic; (D) PTEN mRNA levels were significantly reduced in DLBC cells with miR-425-5p mimic; (E) miR-425-5p and PTEN were negatively correlated in DLBC tissue. Data: mean \pm SD. ${ }^{* *}, \mathrm{P}<0.01$; ${ }^{* * *}, \mathrm{P}<0.001$. DLBC, diffuse large B-cell lymphoma; mRNA, messenger RNA.

U2932-WT, mimic NC vs. miR-425-5p mimic: $1.000 \pm 0.235$ vs. $0.359 \pm 0.138, \mathrm{P}<0.001$, Figure $3 B, 3 C)$. However, little effect was observed on mutated fragments of PTEN 3'-UTR (Figure 3B,3C). PTEN transcript levels were lower in miR425-5p mimics-transfected cells (TMD8, mimic NC vs. miR-425-5p mimic: $1.000 \pm 0.241$ vs. $0.358 \pm 0.142, \mathrm{P}<0.001$; U2932, mimic NC vs. miR-425-5p mimic: $1.000 \pm 0.292$ vs. $0.468 \pm 0.0829, \mathrm{P}<0.001$, Figure $3 D$ ). Furthermore, miR-425$5 \mathrm{p}$ also negatively regulated the related PTEN transcript levels in DLBC tissue $\left(\mathrm{P}=0.035, \mathrm{R}^{2}=0.178\right.$, Figure $\left.3 E\right)$. As a result, PTEN may be indicated as a target of miR-425-5p in the cells.

\section{miR-425-5p promotes DLBC via the PTEN/PI3K pathway}

We analyzed the expression profiles of miR-425-5p mimic cells by assessing the PTEN mRNA levels (Figure 4A), to suggest that blocking of the said receptor can decrease cellular proliferation and oncogenic development. It could also be observed that miR-425-5p could enhance cell proliferation, evident by the increased cellular viability. Moreover, the same can be reversed by transfecting the cells with PTEN (Figure 4B,4C). Additionally, cells obtained from DLBC tissues were treated with PI3K inhibitor LY294002 + miR-425-5p or LY294002 mimics (Figure 4D).

Meanwhile, we found that the activity of PI3K was significantly reduced in transfected cells than in mimic control $(\mathrm{P}<0.01)$. The activity of PI3K in DLBC cells transfected with miR-425-5p mimic and LY294002 was high in comparison with the other counterpart (i.e., LY294002) $(\mathrm{P}<0.01)$. Thus, it can further be suggested that PTEN/ $\mathrm{PI} 3 \mathrm{k}$ signaling is associated with the pro-tumorigenic effect 
A
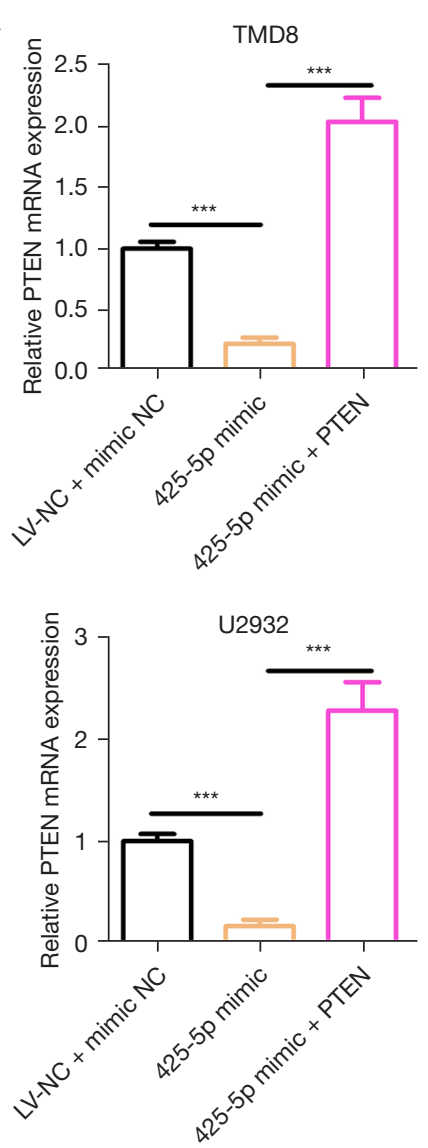

B

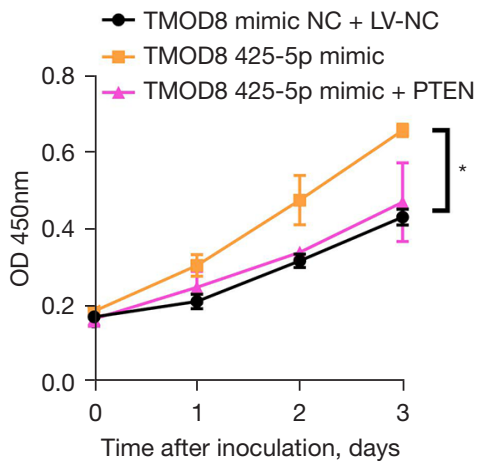

D

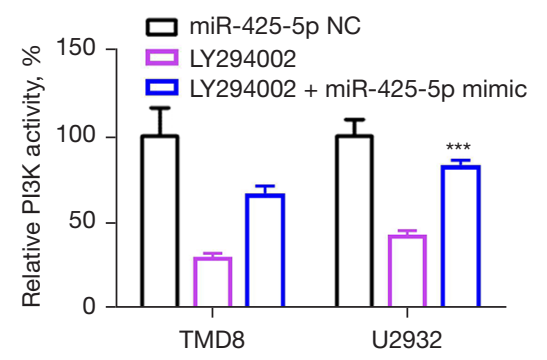

C

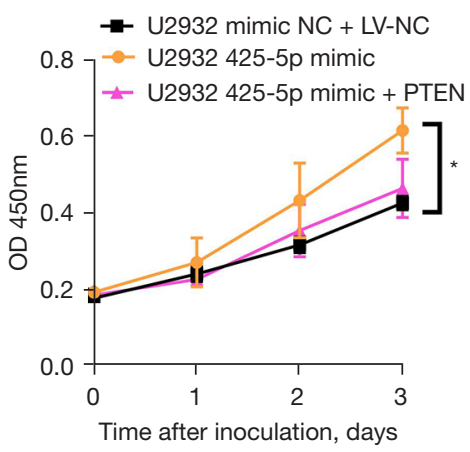

$E$

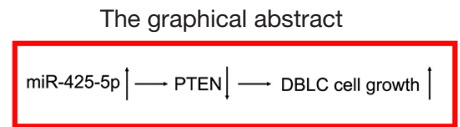

Figure 4 Effects of miR-425-5p on PTEN/PI3K. (A) qRT-PCR analysis of PTEN expression; (B,C) CCK-8 analysis in indicated DLBC cell lines; (D) the PI3K kinase activity was determined in indicated DLBC cells; (E) schematic diagram of miR-425-5p-PTEN/PI3K axis in enhancing diffuse large B cell lymphoma growth. Data: mean \pm SD. *, $\mathrm{P}<0.05$; ${ }^{* *}, \mathrm{P}<0.001$. DLBC, diffuse large B-cell lymphoma; qRTPCR, real-time reverse transcription-PCR.

of miR-425-5P.

\section{Discussion}

In this regard, miRNA has been identified to be one of the key players in regulating oncogenic developments, and therefore may possibly be manipulated in order to identify effective therapeutic outcomes (17-21). Other studies have supported the above-mentioned suggestion, including Sun et al., who confirmed the reduced progression of DLBC post-inhibition of miR-214, which was achieved by blocking DLBC (22). Additionally, Sun et al. also suggested that miR$223-3 p$ is responsible for the increased rate of proliferation of cells in DLBC through PPP2R2A regulation (23). Recently, studies have already confirmed that circulating miR-155 is responsible for poor prognosis and reduced survival (24). These studies have effectively confirmed the role of miRNAs as efficient biomarkers for cancer prognosis, including DLBC. In all of the relevant studies proposed herein, the function of miR-425-5p has not yet been explored, highlighting the need for special attention. Therefore, in the current work, we examined the inherent mode of DLBC cell progression induced by miR-425-5p (Figure 4E).

In the present work, we established the significance of miR-425-5p inhibition as being responsible for the reduced progression of DLBC. The proposed biomarker (i.e. miR-425-5p) is reportedly responsible for targeting multiple genes, including but not limited to Dickkopf 3 (25), CYLD (26), GSK3ß (27), AIFM1 (28), and PTCH1 (29), and was therefore used as a basis for targeting PTEN as the potential therapeutic module. The study further allowed 
us to confirm that cells obtained from DLBC tissues can be transfected using luciferase reporter assay for targeting PTEN-3'UTR, which may functionally lead to the inhibition of miR-425-5p.

We also carried out reporter assays using luciferase, and observed that PTEN-3'UTR may be directly targeted by miR-425. Further investigation confirmed that targeting cells with PTEN could reduce the cancer-causing effect of miR-425-5p via the PTEN/PI3K pathways. PTEN serves as a suppressor of tumors, which has well-defined phosphatase activity (30). It facilitates the progression of the cell cycle, apoptosis inhibition, and has been shown to be highly active in various human cancers $(31,32)$. PI3K signaling can be suppressed by PTEN, which also exhibits anti-cancer functions (33). The examination of the biochemical mode of action of miR-425-5p in DLBC cells, and its cancer-promoting effects were found to be carried out via PTEN/PI3K signaling axis manipulation. Since PI3K/AKT signaling activates downstream of the mammalian target of rapamycin (mTOR) pathway, which is implicated in autoimmune and infectious diseases such as human immunodeficiency virus (HIV) and Sars-COV2 (34-42), it can be speculated that miR-425-5p may also play a pathogenic role in other conditions such as multiple sclerosis, breast cancer, and HIV infection. These are worthy of further study in the future. However, the present study has some limitations that should be noted, including (I) the lack of overexpression experiments in normal cells, (II) the lack of AKT inhibitor experiments to verify the effect of mir-425-5p on the PTEN/PI3K signaling pathway, (III) lack of the effects of miR-425-5p by in vivo experiments, (IV) validate the target gene of miR-425-5p by rescue experiments, $(\mathrm{V})$ the effects of miR-425-5p on apoptosis, We will in future research to address these deficiencies indepth exploration.

In conclusion, we found that miR-425-5p plays an oncogenic role in DLBC and facilitates an oncogenic phenotype via the inhibition of PTEN. These results add to our existing knowledge on the function of miR-425$5 \mathrm{p}$ and also present new therapeutic schemes for DLBC detection and therapy. Further experiments on angiogenesis and metastasis in DLBC will contribute to elucidating the functions of miR-425-5p.

\section{Acknowledgments}

Funding: None.

\section{Footnote}

Reporting Checklist: The authors have completed the MDAR reporting checklist. Available at https://dx.doi. org/10.21037/tcr-21-2394

Data Sharing Statement: Available at https://dx.doi. org/10.21037/tcr-21-2394

Conflicts of Interest: All authors have completed the ICMJE uniform disclosure form (available at https://dx.doi. org/10.21037/tcr-21-2394). The authors have no conflicts of interest to declare.

Ethical Statement: The authors are accountable for all aspects of the work in ensuring that questions related to the accuracy or integrity of any part of the work are appropriately investigated and resolved. This study was approved by the institutional review board of First Hospital Affiliated to Fujian Medical University. All participants were informed about the outcome and provided written informed consent, and participants identity was also kept hidden.

Open Access Statement: This is an Open Access article distributed in accordance with the Creative Commons Attribution-NonCommercial-NoDerivs 4.0 International License (CC BY-NC-ND 4.0), which permits the noncommercial replication and distribution of the article with the strict proviso that no changes or edits are made and the original work is properly cited (including links to both the formal publication through the relevant DOI and the license). See: https://creativecommons.org/licenses/by-nc-nd/4.0/.

\section{References}

1. Alizadeh AA, Eisen MB, Davis RE, et al. Distinct types of diffuse large B-cell lymphoma identified by gene expression profiling. Nature 2000;403:503-11.

2. Coutinho R, Clear AJ, Owen A, et al. Poor concordance among nine immunohistochemistry classifiers of cell-oforigin for diffuse large B-cell lymphoma: implications for therapeutic strategies. Clin Cancer Res 2013;19:6686-95.

3. Veldman-Jones MH, Lai Z, Wappett $M$, et al. Reproducible, Quantitative, and Flexible Molecular Subtyping of Clinical DLBCL Samples Using the NanoString nCounter System. Clin Cancer Res 2015;21:2367-78. 
4. Hans CP, Weisenburger DD, Greiner TC, et al. Confirmation of the molecular classification of diffuse large B-cell lymphoma by immunohistochemistry using a tissue microarray. Blood 2004;103:275-82.

5. Nosrati A, Monabati A, Sadeghipour A, et al. MYC, BCL2, and BCL6 rearrangements in primary central nervous system lymphoma of large B cell type. Ann Hematol 2019;98:169-73.

6. Zhang TJ, Wang YX, Yang DQ, et al. Down-Regulation of miR-186 Correlates with Poor Survival in de novo Acute Myeloid Leukemia. Clin Lab 2016;62:113-20.

7. Shen Z, Gu X, Mao W, et al. Characterization of microRNA expression profiles by deep sequencing of small RNA libraries in leukemia patients from Naxi ethnic. Transl Cancer Res 2019;8:160-9.

8. Lin H, Huang ZP, Liu J, et al. MiR-494-3p promotes PI3K/AKT pathway hyperactivation and human hepatocellular carcinoma progression by targeting PTEN. Sci Rep 2018;8:10461.

9. Huang $\mathrm{H}, \mathrm{Xu} \mathrm{Y}$, Guo Z, et al. MicroRNA-133b inhibits cell proliferation and promotes apoptosis by targeting cullin 4B in esophageal squamous cell carcinoma. Exp Ther Med 2018;15:3743-50.

10. Zhao L, Liu Y, Zhang J, et al. LncRNA SNHG14/miR5590-3p/ZEB1 positive feedback loop promoted diffuse large B cell lymphoma progression and immune evasion through regulating PD-1/PD-L1 checkpoint. Cell Death Dis 2019;10:731.

11. Fang F, Song T, Zhang T, et al. MiR-425-5p promotes invasion and metastasis of hepatocellular carcinoma cells through SCAI-mediated dysregulation of multiple signaling pathways. Oncotarget 2017;8:31745-57.

12. Cristóbal I, Madoz-Gúrpide J, Rojo F, et al. Potential therapeutic value of miR-425-5p in metastatic colorectal cancer. J Cell Mol Med 2016;20:2213-4.

13. Zhang $Z$, Wen $M$, Guo J, et al. Clinical value of miR-425-5p detection and its association with cell proliferation and apoptosis of gastric cancer. Pathol Res Pract 2017;213:929-37.

14. Xiao S, Zhu H, Luo J, et al. miR-425-5p is associated with poor prognosis in patients with breast cancer and promotes cancer cell progression by targeting PTEN. Oncol Rep 2019;42:2550-60.

15. Zhou JS, Yang ZS, Cheng SY, et al. miRNA-425-5p enhances lung cancer growth via the PTEN/PI3K/AKT signaling axis. BMC Pulm Med 2020;20:223.

16. Abubaker J, Bavi PP, Al-Harbi S, et al. PIK3CA mutations are mutually exclusive with PTEN loss in diffuse large
B-cell lymphoma. Leukemia 2007;21:2368-70.

17. Hu YR, Xing SL, Chen C, et al. Tiaoxin Recipe, a Chinese herbal formula, inhibits microRNA-34a expression in the APPswe/PS1 $\triangle \mathrm{E} 9$ mouse model of Alzheimer's disease. J Integr Med 2019;17:404-9.

18. Zhou H, Yang L, Xu X, et al. miR-34a inhibits esophageal squamous cell carcinoma progression via regulation of FOXM1. Oncol Lett 2019;17:706-12.

19. Li Y, Chen D, Gao X, et al. LncRNA NEAT1 Regulates Cell Viability and Invasion in Esophageal Squamous Cell Carcinoma through the miR-129/CTBP2 Axis. Dis Markers 2017;2017:5314649.

20. Jia $\mathrm{Y}, \mathrm{Lu} \mathrm{H}$, Wang C, et al. miR-25 is upregulated before the occurrence of esophageal squamous cell carcinoma. Am J Transl Res 2017;9:4458-69.

21. Zheng TL, Li DP, He ZF, et al. miR-145 sensitizes esophageal squamous cell carcinoma to cisplatin through directly inhibiting PI3K/AKT signaling pathway. Cancer Cell Int 2019;19:250.

22. Sun JR, Zhang X, Zhang Y. MiR-214 prevents the progression of diffuse large B-cell lymphoma by targeting PD-L1. Cell Mol Biol Lett 2019;24:68.

23. Sun S, Wang H, Ji M. Overexpression of miR222-3p Promotes the Proliferation and Inhibits the Apoptosis of Diffuse Large B-Cell Lymphoma Cells via Suppressing PPP2R2A. Technol Cancer Res Treat 2019;18:1533033819892256.

24. Ahmadvand M, Eskandari M, Pashaiefar H, et al. Over expression of circulating miR-155 predicts prognosis in diffuse large B-cell lymphoma. Leuk Res 2018;70:45-8.

25. Ning JZ, Yu WM, Cheng F, et al. MiR-425 Promotes Migration and Invasion in Bladder Cancer by Targeting Dickkopf 3. J Cancer 2020;11:3424-32.

26. Yan YF, Gong FM, Wang BS, et al. MiR-425-5p promotes tumor progression via modulation of CYLD in gastric cancer. Eur Rev Med Pharmacol Sci 2017;21:2130-6.

27. Liu S, Wang Q, Liu Y, et al. miR-425-5p suppresses tumorigenesis and DDP resistance in human-prostate cancer by targeting GSK3 $\beta$ and inactivating the Wnt/ $\beta$-catenin signaling pathway. J Biosci 2019.

28. Zhang Y, Yang Y, Liu R, et al. Downregulation of microRNA-425-5p suppresses cervical cancer tumorigenesis by targeting AIFM1. Exp Ther Med 2019;17:4032-8.

29. Yuan Z, Xiu C, Liu D, et al. Long noncoding RNA LINCPINT regulates laryngeal carcinoma cell stemness and chemoresistance through miR-425-5p/PTCH1/SHH axis. J Cell Physiol 2019;234:23111-22. 
30. Li DM, Sun H. PTEN/MMAC1/TEP1 suppresses the tumorigenicity and induces G1 cell cycle arrest in human glioblastoma cells. Proc Natl Acad Sci U S A 1998;95:15406-11.

31. Bellacosa A, Chan TO, Ahmed NN, et al. Akt activation by growth factors is a multiple-step process: the role of the PH domain. Oncogene 1998;17:313-25.

32. Li JW, Wang XY, Zhang X, et al. (-)-Epicatechin protects against myocardial ischemia-induced cardiac injury via activation of the PTEN/PI3K/AKT pathway. Mol Med Rep 2018;17:8300-8.

33. Qin Y, Huo Z, Song X, et al. mir-106a regulates cell proliferation and apoptosis of colon cancer cells through targeting the PTEN/PI3K/AKT signaling pathway. Oncol Lett 2018;15:3197-201.

34. Donia M, Mangano K, Amoroso A, et al. Treatment with rapamycin ameliorates clinical and histological signs of protracted relapsing experimental allergic encephalomyelitis in Dark Agouti rats and induces expansion of peripheral CD4+CD25+Foxp3+ regulatory $\mathrm{T}$ cells. J Autoimmun 2009;33:135-40.

35. Mammana S, Bramanti P, Mazzon E, et al. Preclinical evaluation of the PI3K/Akt/mTOR pathway in animal models of multiple sclerosis. Oncotarget 2018;9:8263-77.

36. Steelman LS, Martelli AM, Cocco L, et al. The therapeutic potential of mTOR inhibitors in breast cancer. Br J Clin

Cite this article as: $\mathrm{Wu} \mathrm{W}$, Chen $\mathrm{L}$, Chen $\mathrm{C}$, Yu L, Zheng J. miRNA-425-5p enhances diffuse large B cell lymphoma growth by targeting PTEN. Transl Cancer Res 2021;10(11):4905-4913. doi: $10.21037 /$ tcr-21-2394
Pharmacol 2016;82:1189-212.

37. Evangelisti C, Evangelisti C, Chiarini F, et al. Therapeutic potential of targeting mTOR in T-cell acute lymphoblastic leukemia (review). Int J Oncol 2014;45:909-18.

38. Matsuda S, Ichimura M, Ogino M, et al. Effective PI3K modulators for improved therapy against malignant tumors and for neuroprotection of brain damage after tumor therapy (Review). Int J Oncol 2016;49:1785-90.

39. Hafsi S, Pezzino FM, Candido S, et al. Gene alterations in the PI3K/PTEN/AKT pathway as a mechanism of drugresistance (review). Int J Oncol 2012;40:639-44.

40. Nicoletti F, Fagone P, Meroni P, et al. mTOR as a multifunctional therapeutic target in HIV infection. Drug Discov Today 2011;16:715-21.

41. Nicoletti F, Lapenta C, Donati S, et al. Inhibition of human immunodeficiency virus (HIV-1) infection in human peripheral blood leucocytes-SCID reconstituted mice by rapamycin. Clin Exp Immunol 2009;155:28-34.

42. Fagone P, Ciurleo R, Lombardo SD, et al. Transcriptional landscape of SARS-CoV-2 infection dismantles pathogenic pathways activated by the virus, proposes unique sexspecific differences and predicts tailored therapeutic strategies. Autoimmun Rev 2020;19:102571.

(English Language Editor: A. Kassem) 